\title{
Reconstruction of the sculpture surface in the process of optical 3D scanning
}

\author{
N. SHCHEGOLEVA ${ }^{1,2}$, V. PARFENOV 3 , S. SMOLINA ${ }^{3}$ \\ ${ }^{1}$ St.Petersburg State University, Saint Petersburg 199034, RUSSIA \\ ${ }^{2}$ Plekhanov Russian University of Economics Stremyanny lane, 36, Moscow, 117997, RUSSIA \\ ${ }^{3}$ St.Petersburg Electrotechnical University, Saint Petersburg 197376, RUSSIA
}

\begin{abstract}
Currently, 3D scanning technology is used for high-precision measurements and fixing the geometric shape of various objects. However, when creating a computer 3D-model as a result of processing the array of data obtained from the scanning process, may contain errors. Errors may related to the features of the studied object (material, weight, size, location), functional properties of the software used or they may be the low qualification of the software engineer involved in processing 3D scan data. The task of this work was a finding the technical solutions, which allows one to reconstruct the objects surface, recorded using 3D scanning in the process of creating their computer 3D models. We proposed to use the inverse distance method, which is used in biometry to improve the quality of reconstruction of the 3D surface of a human face. Experiments have shown that the result of the reconstruction makes it possible to increase the accuracy of creating 3D models by recovering gaps in the surface of an object that were made during the 3D scanning process. The proposed approach allows one to construct a 3D surface without solving the triangulation problem, which leads to a reduction in computational costs.
\end{abstract}

Key-Words: - sculpture 3D scanning, reconstruction of the surface, inverse distance method, restoring missing measurements, electronic passports of monuments

Received: August 3, 2019. Revised: February 8, 2020. Accepted: February 3, 2020. Published: March 5, 2020.

\section{Introduction}

Laser and optical 3D scanning technologies came to the museum area in the mid of 1990s. This made it possible to approach the task of documenting monuments of history and culture at a higher (as compared with the traditionally used photography) scientific and technical level. 3D scanning allows one not only to capture a three-dimensional image of an object, but also provides highly accurate information about its size and geometrical features of surface shape [1], [2]. As a result of scanning, a so-called cloud of point is created - a computer 3Dmodel file in one of the graphic formats (usually STL (Stereolithography) or OBJ (3D Object)), it can be easily exported to all major standards for exchanging 3D data for further processing. This allows us to consider 3D models as high-precision electronic passports of monuments. Such a passport contains information that may be indispensable in case of damage or loss of monuments as a result of natural disasters or vandal attacks. 3D models can be used to create computer animations, for example, historical reconstructions of palaces, park ensembles, streets, etc. [3].
Museums use an automated turntable for object scanning. However, it can only be used when working with small weight objects (usually up to 200-300 kg). However, if we are dealing with bronze or marble statues 2.5-3 meters high and even more so installed on city streets, in parks, etc., the use of turntables is not possible. When scanning large objects, the amount of scans (with the same angle shots at different angles and with different heights) can be up to 300-500 scans. With such a number of scans during shooting, you may simply not notice that one of the scans has a section where a flare has formed (or, on the contrary, there was a surface shadowing during the shooting).

It should be noted that, the accuracy of creating 3D models can also be reduced due to the loss of information about separate surface areas of the scanned objects as a result of bright light highlights or, on the contrary, insufficient surface illumination during the scanning process. In such cases, when creating 3D-models, these "dead zones" on the surface of the object are usually filled with the help of the so-called "sculpting" when working in specialized programs designed for processing 3D scanning data. In fact, we are talking about the 
"manual" refinement of 3D models using computer graphics techniques. However, it is obvious that such ways of refining 3D-models introduce an element of subjectivity in the process of documenting registered objects, which casts doubt on the adequacy of the resulting 3D-models to the original objects.

Software for creating a computer 3D-models are based on triangulation procedures with straight-line interpolation. It should be noted that the triangulation and calculation of the connected coordinates produce very good representations of surfaces. However, this is achieved by use of a very large number of triangles in the original grid, which inevitably leads to high computational costs. But more importantly, triangulation does not allow restoring missing measurements, which leads to the appearance of the so-called "black holes" on the surface of objects. It should be noted that there are various modifications of triangulation to reduce computational complexity, for example adaptive triangulation; however, they do not solve the problem of gaps filling. Elimination of such gaps is usually performed manually using spline interpolation.

Another source of errors may be insufficiently high qualification of the software engineer involved in processing $3 \mathrm{D}$ scan data. The latter is due to the fact that the highest accuracy of creating 3D models can be achieved by processing the scanned data not in an automatic, but so-called "manual" mode. But this inevitably introduces a "human factor" into the process of creating 3D models [4]. For this reason, at the moment the task of finding mathematical methods that can eliminate the human factor in the processing of 3D scanning data and improve the accuracy of creating 3D models is very important.

\section{Method of inverse distances}

At the moment there is no general approach to solve this problem. Existing methods can be divided into three categories [5]: methods based on polygonal representation (restores arbitrary holes and irregular data can be used, but requires significant computational resources and user involvement in processing), methods based on parametric representation (does not require user involvement in the formation of the model but allows you to repair only small holes, requires holes to be regular and it does not work well with highly curved surfaces), methods based on signed distance functions and voluminous performances (restores arbitrary holes, irregular data can be used, restores large holes and does not require user input but requires significant computational resources and high resolution input). Professional software packages (RapidForm XOR, RapidForm XOR, etc.) provide an opportunity to reconstruct holes automatically or manually. However, these software packages require a large amount of resources, even to restore the most simple models. This is due to the fact that most of them use triangulation.

For this reason, at the moment the task of finding mathematical methods that can eliminate the human factor in the processing of 3D scanning data and improve the accuracy of creating 3D models is very important. The development of such a method is the purpose of this work. The analysis of the scientific literature carried out by the authors showed that no one has used such methods so far. It allows us to speak about the scientific novelty and original character of the results obtained in our work.

To restore the surface of sculptures we used a method of inverse distances that which can be attributed to the third category. It is used in biometry to improve the quality of reconstruction of the 3D surface of a human face. This method allows us not to solve the problem of triangulation. Instead, it is possible to construct the surface of the object from the measurements obtained, belonging to an irregular grid of measurements. A detailed description of the method can be found in [6].

The input information is a cloud of points. Each point is described by its coordinates $\left\{x_{l}, y_{l}, z_{l}\right\}$, where $x$ and $\mathrm{y}$ determine the coordinates of the point, and $z$ is the height of the point relative to the XY plane. We obtained $L$ points from initial data cloud by using a random uniformly distributed number generator. Algorithm for reconstruction of the 3D surface consists of two stages: model construction and transfer the source data onto a regular grid of $M \times N$ size.

We solve the problem of data translation from an irregular grid to a regular one in 2 steps.

1. The initial information for the first step includes set points $\left\{x_{l}, y_{l}, z_{l}\right\}$, recorded in corresponding vectors $X, Y, Z, L \times 1$-size each.

The aim of this step is a construction of a model $z_{l}=f\left(x_{l}, y_{l}\right)$, that represents the dependence of $z_{l}$ from $\left\{x_{l}, y_{l}\right\}$. To reach the aim we make the following steps.

1.1. Let us represent all coordinates $\left\{x_{l}, y_{l}\right\}$ of the initial data with a vector $K$ in a complex form in the following way:

$$
K=X+j Y \text {, где } j=\sqrt{-1}
$$

and it is assumed that the vector of $\mathrm{K}$ do not contain data that $k_{l} \neq k_{d}$ for $l \neq d$. And this condition of "data 
non-multiplicity" is the only one in solving of the given task.

1.2. Now create a matrix $K$, repeating $L$ times the resulting vector $\mathrm{K}$ :

$$
\boldsymbol{K}=[K K \ldots K],
$$

where the matrix $\boldsymbol{K}$ is the size $L \times L$.

1.3. Compute the distance between all the claims of irregular grid:

$$
\boldsymbol{D}=\operatorname{abs}\left(\boldsymbol{K}-\boldsymbol{K}^{\mathrm{T}}\right),
$$

where the matrix $\boldsymbol{D}$ is the size $L \times L$.

Note that only when the condition "nonrepeating data", the rank of $\boldsymbol{D}$ is equal to its order. Only in this case, a transition to the next step of the algorithm.

1. Compute the vector of regression parameters $B$ such that:

$$
B=D^{-1} Z \text {, }
$$

where the vector $B$ has a size $L \times 1$.

2. The initial information to the second step are:

- Set points $\left\{x_{l}, y_{l}, z_{l}\right\}$, recorded in the corresponding vectors $X, Y, Z$;

- vector $B$ regression parameters;

- the values of $L, M$ and $N$.

The aim of the second step is initial data (cloud) transfer onto a regular grid $M \times N$-size, and as a result we will receive a "range image". The calculation of each value $I(m, n)$ of the "range image" is realized in the following way:

$$
z_{q l}=\sum_{j=1}^{N} b_{j} *\left|k_{q l}-k_{j}\right|,
$$

$m=1,2, \ldots, M$ and $n=1,2, \ldots, N$.

where: $k(n, m)=x_{n}+j y_{m}, \forall n=1,2, \ldots, N$ and $m=1,2, \ldots, M$;

$\left|k_{q l}-k_{j}\right|$ - modulus of difference between the new and old coordinates;

$$
\begin{aligned}
& x_{n}=\min (x)+n(\max (x)-\min (x)) /(N-1) ; \\
& y_{m}=\min (y)+m(\max (y)-\min (y)) /(M-1) ; \\
& k_{l}=x_{l}+j y_{l}
\end{aligned}
$$

Note that the solution (2) has also been achieved in the condition of "nonrepeating data", which can be written as follows: $\left|k(n, m)-k_{l}\right| \neq 0, \forall l \neq n$ и $l \neq m$.

Implementation algorithm (2) is given below.

2.1. For set $\left\{x_{l}, y_{l}\right\}$, recorded in the corresponding vectors $X$ and $Y$, we calculate the coordinates of the boundary so that:

$$
\begin{aligned}
& \operatorname{Max} \_\mathrm{Y}=\max (Y) ; \operatorname{Min} \_\mathrm{Y}=\min (Y) \text {; } \\
& \operatorname{Max} \_\mathrm{X}=\max (X) ; \operatorname{Min} \_\mathrm{X}=\min (\mathrm{X}) \text {. }
\end{aligned}
$$

2.2. Then calculate the sampling interval of a regular grid with considering the size of $M$ and $N$ «range image»:

$$
\text { delta } Y=\left(\max \_Y-\min \_Y\right) /(\mathrm{M}-1) \text {; }
$$

$\operatorname{delta} X=\left(\max \_X-\min \_\mathrm{X}\right) /(\mathrm{N}-1)$.

2.3. Now define all the coordinates of a regular grid for a given size «range image» and write them in the corresponding vectors:

$$
\begin{aligned}
X_{r} & =\left(\min \_X: d e l t a X: m a x \_X\right) ; \\
Y_{r} & =\left(\text { min_Y:delta } Y: m a x \_Y\right) .
\end{aligned}
$$

2.4. Create a "zero" work array size $M \times N$ for «range image»:

$$
I=[0]_{M \times N}
$$

2.5. For all the current coordinates of $m=1,2, \ldots$, $M$ and $n=1,2, \ldots, N$ form the vector $X_{Y}$, consisting of $L$ rows of coordinates $[X r(n)+j Y r(m)]$, so that:

$$
X_{Y}=\left[\begin{array}{l}
X r(n)+j \operatorname{Yr}(m) \\
\operatorname{Xr}(n)+j \operatorname{Yr}(m) \\
\cdots \\
\operatorname{Xr}(n)+j \operatorname{Yr}(m)
\end{array}\right] .
$$

2.6. Calculate the distance between the new and the original coordinates

$$
D_{\text {new }}=\operatorname{abs}\left(X_{Y}-K\right) \text {. }
$$

2.7. Calculate the values of «range image» for the pixel $(m, n)$ :

$$
I(m, n)=D_{n e w}^{T} B
$$

and then proceed to the selection of a new current value of $m$ and $n$, and repeat steps $(3) \div(5)$.

\section{Experiments}

It would seem that this method can be immediately applied to scans of sculptures. However, several problems were identified. We illustrate this with experiments.

To solve the problem of recovering missing measurement when constructing 3D models of objects an application was developed in the Matlab environment. The graphical user interface is created in the App Designer editor. The application allows you to create or load a point cloud, restore the surface and save the 3D model in Matlab-format or in stl-format. Typically, when shooting an object, multiple 3D-scans are performed. Obviously, the most accurate result can be obtained only by joint processing of all 3D-scans, however, the quality of each individual 3D-scan affects the quality of the resulting model. Therefore, the object of study in the experiment is a separate 3D-scan.

In each experiment, 3D models with measurement gaps were used. The result of each experiment is a 3D model in stl-format. For the first experiment, the face of the sculpture was taken with measurement gaps not exceeding 3\% of the entire surface. The fig. 1 shows input scan and fig. 2 shows reconstruction of the model with measurement omissions less than $3 \%$. It is clearly 
seen that there are no measurement omissions in the model, surface irregularities are smoothed. Fig. 3 demonstrated the symmetry lines of the original sculpture and the restored model, which are almost exactly the same everywhere, except for emissions in the source data, which are shown in blue. The standard deviation is 0.13 .

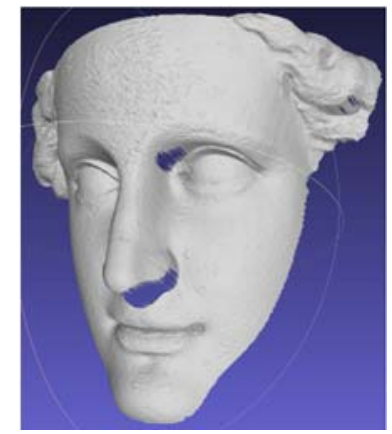

Fig. 1. Input scan

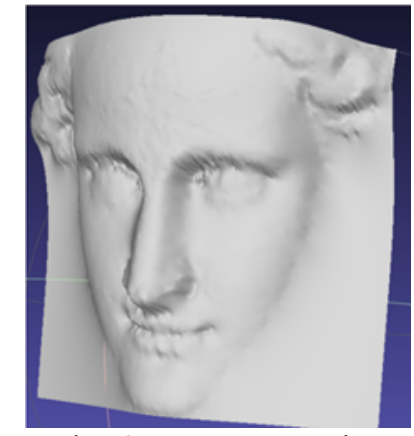

Fig. 2. Reconstruction of the model

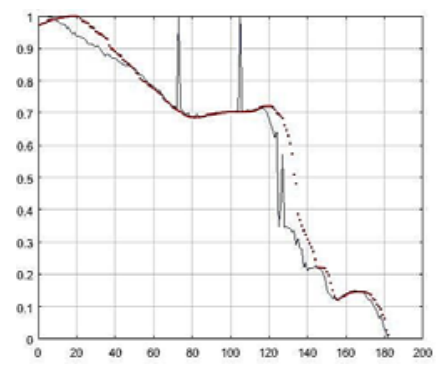

Fig. 3. Symmetry lines of the original sculpture and the restored model

For the second experiment, a 3D model of a turtle sculpture was taken. Damage is more than $30 \%$ of the model's surface. The fig. 4 shows input scan and fig. 5 shows restoration of the model with measurement omissions greater than $3 \%$. In the turtle scan $30 \%$ of measurements are missing. Even such significant omissions on the turtle's leg are completed in the form of a regular rounded surface. The value of the standard deviation is 0.1638 .

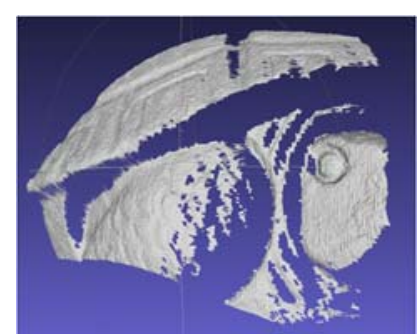

fig. 4. Turtle scan

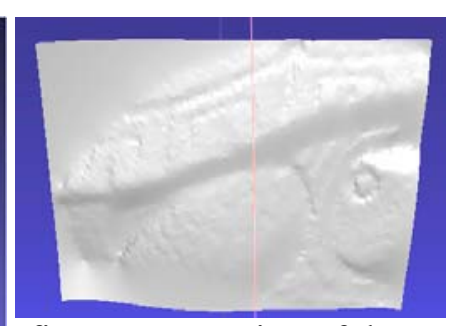

fig. 5. Restoration of the model with $30 \%$ measurement omissions
The result of experiments showed that this method has the following advantages: namely it is able to work with irregular data, to restore measurement skips more then $30 \%$, and to recover free form skips, does not require high resolution input data, able to restore curved surfaces, does not require solving the problem of triangulation to recover the gaps, which reduces the computational complexity and time.

Another experiment was carried out to restore the sculpture, the dimensions of which are much larger than in experiments 1 and 2.

The fig. 6 shows scan of the profile sculpture of a man, dimensions of which are larger than previously considered $(15000)$. On the head there are large curls, on which there are holes. Such holes are typical for models of sculptures. The model has been reconstructed (fig. 7), but there are inaccuracies, the standard deviation is 0.2 and it is larger than in previous experiments, which allows to conclude that the larger the model, the lower the quality.

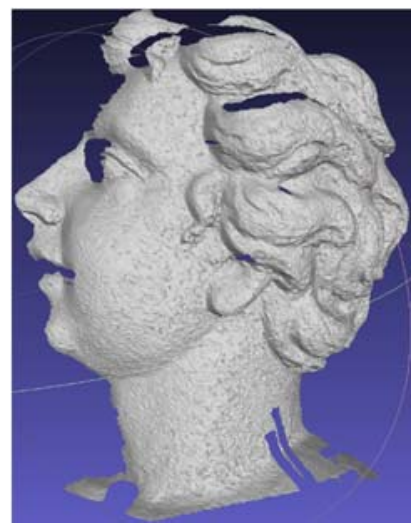

fig. 6. Scan of the profile sculpture

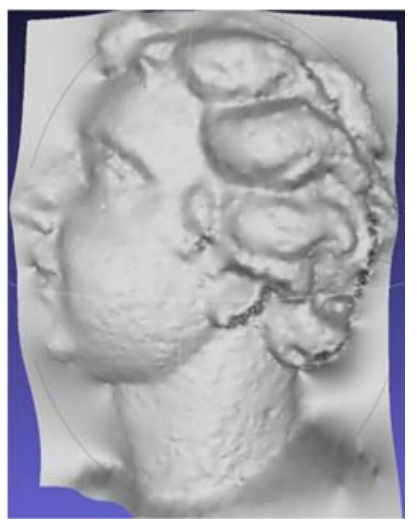

fig. 7. Reconstruction of the model
To test this hypothesis, a fragment of the scan was taken (fig. 8) and built a model of this fragment (fig.10). A comparison of the pairs of figures 9 and 10 shows that when restoring a fragment of the sculpture scan, the quality became much better. This is also confirmed by the standard deviation, which is 0.1289 in comparison with the result of the first experiment, 0.1397 . In fig. 10 presents a 3D model in stl-format, where also the details of the eye became sharper.

It should be noted that the algorithm considered above was used to build a 3D model using only one scan, however, it can be applied to all sculpture scans (as a rule, they are made on the order of several hundreds). Since its computational complexity is significantly lower compared to triangulation, this will reduce the processing time of scans and their joint use will allow building a highquality sculpture model. 
The most effective method for improving the quality of a 3D model is to divide the model into fragments and restore each of them individually. This means that this algorithm allows you to restore the model to almost the selected level of detail.

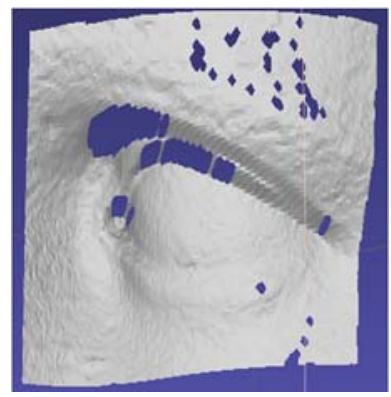

fig. 8. Fragment of the scan

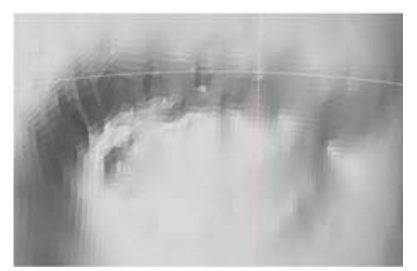

fig. 9. Fragment of model from experiment 1

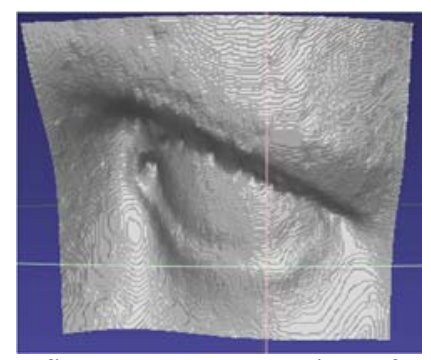

fig. 10. Reconstruction of the scan fragment

\section{Conclusion}

Laser and optical 3D scanning technologies made possible to solve the task of documenting monuments of history and culture. However, computer 3D-model based on this scans may contain errors, which appear due to omissions of measurements from the scanner caused by various reasons. The experiments demonstrated that the result of reconstruction does not contain the amount or quality of black holes that has the original object. The method used has the following advantages: able to work with irregular data; recover significant gaps in measurements of an irregular shape of a curved surface. Using this method allows us not to solve the triangulation task, which leads to a reduction of computational costs. The proposed approach allows us to improve the quality of a reconstructed 3D surface of an object or its parts using the inverse distance method. Therefore this approach can be applied to the restoration of 3D models of art objects (monuments, sculptures, etc.).

\section{References:}

[1] J.-A. Beraldin, F. Blais, L. Cournoyer et al. Portable digital 3-D imaging system for remote sites // Proceedings of IEEE Intern. Symp. on Circuits and Systems, 1998. Vol. 5. P. V-488V-493.

[2] Fontana R., Gambino M. C., Greco M. et al. High-resolution 3D digital models of artworks // Proceedings of SPIE, 2003. Vol. 5146. P. 3443.

[3] V. A. Parfenov, O. V. Frank-Kamenetskaya, I. A. Leonova, S. L. Moshkina. Use of laser 3D scanning for monitoring of sculptural monuments // Izvesitya Sankt- Petersburgskogo gosudarstvennogo elektotekhnicheskogo universiteta. 2018. № 3. p. 73-79.

[4] Vadim Parfenov "Use of 3D laser scanning for documentation, digital reconstruction and physical replication of sculptural monuments (Conference Presentation)", Proc. SPIE 11058, Optics for Arts, Architecture, and Archaeology VII, $110580 \mathrm{~K} \quad$ (22 July 2019); https://doi.org/10.1117/12.2526163

[5] Pérez E. et al. A comparison of hole-filling methods in 3D //International Journal of Applied Mathematics and Computer Science. 2016. - T. 26. - №. 4. - C. 885-903.

[6] Shchegoleva N. L. Facial surface reconstruction in 3D format // JTACS Journal of Theoretical and Applied Computer Science, 2012. - Vol.6, № 4. - P. 37-50. 